\title{
Nucleosynthesis by accelerated particles to account for the surface composition of HD 101065
}

\author{
S. Goriely \\ Institut d'Astronomie et d'Astrophysique, Université Libre de Bruxelles, CP 226, 1050 Brussels, Belgium \\ e-mail: sgoriely@astro.ulb.ac.be
}

Received 17 October 2006 / Accepted 2 January 2007

\begin{abstract}
Context. Recent observations have suggested the presence of radioactive elements, such as Tc, Pm, and $84 \leq Z \leq 99$ elements at the surface of the chemically-peculiar magnetic star HD 101065, also known as Przybylski's star. The peculiar $35<Z<82$ abundance pattern of HD 101065 has been explained so far by diffusion processes in the stellar envelope. However, those processes cannot be called on to explain the origin of short-lived radioelements.

Aims. The large magnetic field observed in Ap stars can be at the origin of a significant acceleration of charged particles, mainly protons and $\alpha$-particles, that in turn can modify the surface content by interaction with the stellar material. This paper explores to what extent an irradiation process resulting from the interaction of the stellar material with energetic particles can by itself account for both the abundances determined by observation on the surface of the chemically peculiar star HD 101065 and the presence of unstable elements.

Methods. Due to the unknown characteristics of the accelerated particles that could be held responsible for this nuclear process, a purely parametric approach is followed, with the proton and $\alpha$-particle flux amplitude and energy distribution taken as free parameters, as well as the total fluence

Results. This kind of irradiation process, at least for high fluences, can lead to a rich nucleosynthesis, including a significant production of $Z>30$ heavy elements, as well as radioelements like Tc and Pm, and even transuranium. In this respect, the energy spectrum of the accelerated particles plays a crucial role. Many observational aspects of HD 101065's composition can be explained quantitatively.

Conclusions. The possible existence of high-fluence irradiation events need to be confirmed by hydrodynamics simulations but, most of all, by spectroscopic observations through detecting short-lived unstable elements on the surface of chemically peculiar stars.
\end{abstract}

Key words. stars: chemically peculiar - nuclear reactions, nucleosynthesis, abundances - stars: individual: HD 101065 acceleration of particles

\section{Introduction}

Chemically peculiar (CP) stars are spectral type A stars that have chemical abundances differing significantly from that of other stars with a similar classification (see Zverko et al. 2004, for a review of the A-star Puzzle). The two major types of CP stars are Am stars that have no detectable magnetic field and Ap stars that have magnetic fields with a large-scale structure. Rapidly oscillating Ap (roAp) stars are a subgroup of magnetic Ap stars that oscillate with non-radial, low-order acoustic $p$-modes with the axis of oscillation aligned with the axis of the magnetic field (Kurtz 1982).

The mechanisms responsible for exciting roAp stars and other physical parameters that distinguish them from nonpulsating CP stars remain an open question. The CP stars exhibit a remarkable variety of elemental enhancements and depletions in their photospheres, sometimes 5 to 6 orders of magnitudes different than found in the sun (Cowley \& Bord 2004). The comparison between roAp and non-roAp stars' surface composition does not reveal large abundance differences. Various scenarios have been suggested to account for the origin of CP stars, including contact binaries that transfer mass to each other and eventually merge into a single star. However, quantitatively, the CP-star abundance peculiarities have been explained almost uniquely on the basis of diffusion processes, i.e. the diffusive segregation of ionic and isotopic species resulting from the balance between radiative and gravitational forces within the atmosphere and subatmospheric regions (Michaud 1970, 2004).

Recent observations suggest the presence of short-lived radioactive elements, such as Tc, Pm, and $84 \leq Z \leq 99$ elements, at the surface of the CP roAp star HD 101065, also known as Przybylski's star (Cowley et al. 2004; Gopka et al. 2004; Bidelman 2005; Gopka et al. 2006). Such a remarkable observation will most certainly need further exploration with scrutiny due to the extremely complicated task of identifying the lines of ionized radioelements in warm, abnormal, stratified, and out-ofthermodynamic-equilibrium atmospheres (Cowley 2005). But if confirmed, it can in no way be explained by diffusion processes. Only nuclear reactions could possibly be responsible for the synthesis of such short-lived radioelements (in particular, Pm's longest isotopic half-life is $17.7 \mathrm{yr}$ ). The large magnetic field observed in Ap stars (in the case of HD 101065, the magnetic field amounts to $B=2300 \mathrm{G}$ ) could be at the origin of a significant acceleration of charged particles, mainly protons and $\alpha$-particles, that in turn can modify the surface content by interaction with the stellar material. More general observations of CP-stars in the $\mathrm{X}$-ray regime show evidence that high energy phenomena are associated with, or even produced by, some CP stars. As shown by Padovani (2004), Ap stars are 3 to 4 times more likely to be associated with X-ray sources than normal A stars, so that 
energetic events can be expected in the vicinity of Ap stars. Due to the success of diffusion, only a few studies back in the $60 \mathrm{~s}$ have been devoted to explaining the CP stars' abundance anomalies by nuclear reaction in the stellar surface layers (see in particular, Brancazio \& Cameron 1967).

The purpose of the present work is to study to what extent the irradiation process resulting from the interaction of the stellar material with energetic particles can alone explain the abundances determined by observation on the surface of the roAp star HD 101065. This includes the particularly large abundances of the heavy elements with $35<Z<82$ by 3 to 4 dex with respect to the solar composition (Cowley et al. 2000), so far explained by diffusion processes, but also the possible presence of the radioactive elements, such as Tc, Pm, and $84 \leq Z \leq 99$ elements, that have been identified (Cowley et al. 2004; Gopka et al. 2004; Bidelman 2005). Detailed spectroscopic analyses have been devoted to HD 101065, leading to the abundance determination for about 55 elements from $\mathrm{Li}$ to $\mathrm{U}$. Even the $\mathrm{Li}$ isotopic ratio ${ }^{6} \mathrm{Li} /{ }^{7} \mathrm{Li}=0.3$ has been derived by Shavrina et al. $(2003,2006)$. Such a wide range of spectroscopic constraints provides an extremely valuable testing ground for theoretical models and justifies our focus here on the abundance composition of HD 101065.

Due to the exploratory nature of the present study, no effort has been made to understand the possible mechanisms that could be held responsible for accelerating the energetic particles. As already discussed, these particles could be locally accelerated, but they could also come from an external source. A purely parametric approach is followed by taking the properties of the accelerated proton and $\alpha$-particle fluxes as free parameters. Orders of magnitude should nevertheless be kept in mind: in the specific case of solar flares typical proton fluences are roughly $10^{17-18} \mathrm{~cm}^{-2}$, while early solar system models call for fluences of about $10^{23} \mathrm{~cm}^{-2}$ to explain the origin of the nowextinct short-lived radionuclides known to have been present in the early solar system (e.g Leya et al. 2003). If HD 101065 is not a T-Tauri star, its putative companion might be one. In addition, the proton fluence can also be expected to be increased by a factor as high as $10^{6}$ if the protons are guided along the magnetic field lines.

It is also worth mentioning that the energetic jet particles from supernova explosions represent another possible extreme scenario in which fluences can be achieved that are higher by orders of magnitude. For example, in bipolar supernova explosions (Maeda \& Nomoto 2003; Fujimoto et al. 2006), we deal, at a radius of $1000 \mathrm{~km}$ from the supernova centre, with fluxes of relativistic jet particles (initially protons that recombine into $\alpha$-particles at a later time during the expansion) that can reach fluences of about $10^{37-40} \mathrm{~cm}^{-2}$ at a speed of about $c / 6$, i.e. a kinetic energy of some $10 \mathrm{MeV}$ per nucleon. The composition of the accelerated particles would also obviously depend on the astrophysics site. Protons and $\alpha$-particles with a typical solar ratio of $\alpha / p=0.1$ seems to be an appropriate guess. However, the absence of accelerated protons could also be expected in some cases, for example in supernova explosion jets where neutrons and protons have recombined into $\alpha$-particles or in a postaccelerated Wolf-Rayet wind.

In Sect. 2, the model used to describe the nucleosynthesis resulting from the interaction of energetic protons and $\alpha$-particles with solar-like material is outlined, including the input physics and the approximation and parametrization adopted. Section 3 is devoted to a relatively broad discussion regarding the nucleosynthesis to be expected for different sets of input parameters. Finally in Sect. 4, the abundance distribution spectroscopically determined in HD 101065 is quantitatively compared with two specific irradiation events. Conclusions are drawn in Sect. 5.

\section{Modelling}

To describe the changes in abundance of the nuclei as a result of the interaction of the energetic incident particles with the lowdensity stellar atmosphere, a nuclear reaction network including all relevant reactions is used. All nuclei with $0 \leq Z \leq 102$ and located between the proton drip line and the neutron-rich side of the valley of stability are included in the network. The chosen set of nuclear species are then coupled by a system of differential equations corresponding to all the reactions affecting each nucleus, i.e. mainly proton, $\alpha$ and neutron captures, $\beta$ and $\alpha$-decays, as well as spontaneous fission decays. The rate of change of the molar fraction $Y_{(Z, A)}$ of a nucleus $(Z, A)$ with charge number $Z$ and mass number $A$ can be written as

$$
\begin{aligned}
\frac{\mathrm{d} Y_{(Z, A)}}{\mathrm{d} t}= & -Y_{(Z, A)} \Phi_{\mathrm{p}}\langle\sigma\rangle_{\mathrm{p}}+\sum_{k} Y_{k} \Phi_{\mathrm{p}}\langle\sigma\rangle_{\mathrm{p}, k}^{(Z, A)} \\
& -Y_{(Z, A)} \Phi_{\alpha}\langle\sigma\rangle_{\alpha}+\sum_{k} Y_{k} \Phi_{\alpha}\langle\sigma\rangle_{\alpha, k}^{(Z, A)} \\
& -Y_{\mathrm{n}} \rho N_{\mathrm{a}}\langle\sigma v\rangle_{\mathrm{n}}^{(Z, A)} Y_{(Z, A)} \\
& +\sum_{k} Y_{\mathrm{n}} \rho N_{\mathrm{a}}\langle\sigma v\rangle_{n, A}^{(Z, A)} Y_{k} \\
& -\lambda_{\beta^{-}}^{(Z, A)} Y_{(Z, A)}-\lambda_{\beta^{+}}^{(Z, A)} Y_{(Z, A)} \\
& +\lambda_{\beta^{-}}^{(Z-1, A)} Y_{(Z-1, A)}+\lambda_{\beta^{+}}^{(Z+1, A)} Y_{(Z+1, A)} \\
& -\lambda_{\alpha}^{(Z, A)} Y_{(Z, A)}+\lambda_{\alpha}^{(Z+2, A+4)} Y_{(Z+2, A+4)} \\
& -\lambda_{\mathrm{sf}}^{(Z, A)} Y_{(Z, A)}+\sum_{k}^{(Z, A)} \lambda_{\mathrm{sf}, k}
\end{aligned}
$$

where $\lambda_{\beta}^{ \pm}, \lambda_{\alpha}$, and $\lambda_{\text {sf }}$ are the $\beta^{ \pm}, \alpha$, and spontaneous fission decay rate, respectively; $N_{\mathrm{a}}$ is the Avogadro number, $\rho$ the local density, and $Y_{\mathrm{n}}$ the neutron abundance. Note that the recycling of a given nucleus by p-induced, $\alpha$-induced, or spontaneous fission into fission products is assumed to take place by symmetric fission to one unique species (the subsequent proton and $\alpha$-captures by the fission products during the irradiation process justify this approximation). in Eq. (1), $\langle\sigma\rangle_{\mathrm{p}}$ and $\langle\sigma\rangle_{\alpha}$ are the total effective cross sections for proton and $\alpha$-captures averaged over the energy distribution $\Phi(E)$, i.e. for the proton case

$\langle\sigma\rangle_{\mathrm{p}}=\frac{1}{\Phi_{\mathrm{p}}} \int_{0}^{\infty} \Phi(E) \sigma_{\mathrm{p}}(E) \mathrm{d} E$

where $\sigma_{\mathrm{p}}(E)$ is the $E$-dependent total proton capture cross section and $\Phi_{\mathrm{p}}=\int_{0}^{\infty} \Phi(E) \mathrm{d} E$ the proton flux amplitude (see below). Similarly, $\langle\sigma\rangle_{\mathrm{p}, k}^{(Z, A)}$ is the partial effective cross section for proton capture on a nucleus $k$ leading to the residual $(Z, A)$ nucleus. The proton and $\alpha$-particle capture cross sections $\sigma(E)$ are either taken from experiments (essentially for light species, Meyer 1972) or estimated with the Talys nuclear reaction code (Koning et al. 2005) which takes all types of direct, pre-equilibrium and compound mechanisms into account to estimate the total reaction probability, as well as the competition between the various open channels. The cross sections are estimated at energies up to $200 \mathrm{MeV}$. The calculation includes single particles (nucleons and alpha), as well as multi-particle emissions and fission. All the experimental information on nuclear masses, deformation, and low-lying states spectra is considered, whenever available. If not, global nuclear level formulas, $\gamma$-ray 
strength functions, and nucleon and $\alpha$-particle optical model potentials are considered to determine the excitation level scheme and the photon and particle transmission coefficients. Due to the large number of open channels at high energies, typically about 30 different ( $\mathrm{p}$ or $\alpha, x \mathrm{n} y \mathrm{pz} \alpha$ ) reaction types corresponding to the emission of $x$ neutrons, $y$ protons, and $z \alpha$-particles need to be taken into account per target nucleus. The reaction network (Eq. (1)) includes about 5000 different species with $0 \leq Z \leq 102$ and some 250000 proton, neutron, and $\alpha$-particle capture reactions. The initial unaffected stellar atmosphere before irradiation is assumed to have a solar composition.

The proton and $\alpha$-particle fluxes are assumed to remain constant in time during the irradiation period $\tau_{\text {irr }}$. Many different energy distributions could be studied. We will restrict ourselves to two types of energy spectra, namely a constant distribution in a restricted $E_{\min } \leq E \leq E_{\max }$ energy range and the widelyused power law introduced by Brancazio \& Cameron (1967) and defined by

$\Phi(E)=K E^{-\gamma}$

over an energy range between $E_{\min }$ and $E_{\max }=200 \mathrm{MeV}$ (here and in the following, $E$ is the kinetic energy per nucleon always expressed in the laboratory system). The constant $K$ is related to the flux amplitudes $\Phi_{\mathrm{p}}$ and $\Phi_{\alpha}$ after normalization of Eq. (3), i.e. $\int_{E_{\min }}^{E_{\max }} \Phi(E) \mathrm{d} E=\Phi_{\mathrm{p}}$ for the proton case. The same energy spectrum is adopted for protons and $\alpha$-particles. Although the power law is a traditional distribution widely used to describe energy spectra of accelerated particles, the energy-constant form might well be closer to reality. Indeed, for a given initial proton energy, due to Coulomb interaction, the energy loss per unit length path is a decreasing function of energy, i.e. $\mathrm{d} E / \mathrm{d} x \propto E^{-1}$, so that lower energy protons see a smaller number of targets per unit loss of energy, with the result that the energy spectrum is rather peaked at the highest initial energies (Clayton et al. 1977).

In the present work, special attention is paid to the role played by the neutrons emitted during the spallation process. The neutron abundance is followed with the same time-dependent equation as in Eq. (1). We assume that the neutrons produced this way are thermalized on much shorter timescales than the typical capture timescales. An upper value for the thermalization time can be estimated by

$$
\tau \simeq \frac{1}{N_{\mathrm{a}} \rho \sigma v} \frac{\sqrt{2}}{\sqrt{2}-1}
$$

where $v$ is the thermal velocity. Assuming the matter to be essentially made of $\mathrm{H}, \sigma$ corresponds to the $\mathrm{H}$-neutron collision cross section, which amounts to about $20 \mathrm{~b}$, so that for a surface temperature of $T \simeq 7000 \mathrm{~K}$ and density $\rho=10^{-7} \mathrm{~g} / \mathrm{cm}^{3}, \tau=2 \mathrm{~s}$, which justifies the fast thermalization of the neutrons for irradiation processes taking place on time scales $\tau_{\text {irr }} \gg \tau$. In this case, the neutron energy spectrum follows a traditional MaxwellBolztmann distribution at a temperature $T$. The neutron capture rates $\langle\sigma v\rangle_{\mathrm{n}}$ are estimated by the Hauser-Feshbach reaction code MOST (Arnould \& Goriely 2006). In Eq. (1), $\langle\sigma v\rangle_{n, k}^{(Z . A)}$ corresponds to either the $(n, \gamma),(n, p)$, or $(n, \alpha)$ reaction rate leading to the formation of the $(Z, A)$ nucleus.

As already discussed in Sect. 1, no effort is made in the present work to explain the possible mechanisms responsible for accelerating the energetic particles. The energy spectrum, the proton and $\alpha$-particle fluxes, and the irradiation time are therefore taken as free parameters. Typical fluxes between $10^{-8}$ and $10^{2} \mathrm{mb}^{-1} \mathrm{~s}^{-1}$ will be considered, leaving more extreme scenarios, such as the one resulting from supernova jets (see above) for future studies. In Sect. 4, we nevertheless estimate the socalled exposure parameter introduced by Brancazio \& Cameron (1967) and corresponding to the time-integrated energy density defined by $w=\langle E\rangle \phi \tau_{\text {irr }} / v$ (where $\langle E\rangle$ is the mean energy per nucleon and $v$ the mean velocity of the accelerated particles) in order to evaluate to what extent the total energy required for the particle acceleration of the event called for to explain the abundances in HD 101065 could be supplied by the magnetic energy.

\section{Results}

We here present the nucleosynthesis resulting from the continuous irradiation of solar-like material by accelerated protons and $\alpha$-particles characterised by a relatively wide range of physical properties. The comparison of such a possible nucleosynthesis with the surface composition of HD 101065 is postponed to Sect. 4.

It can be expected that in the case of relatively low-amplitude fluxes, the proton- and $\alpha$-captures are slower than the $\beta^{+}$decays, so that the nuclear flow follows a path along the valley of $\beta$-stability on the neutron-deficient side. For higher values of $\Phi_{\mathrm{p}}$ and $\Phi_{\alpha}$, neutron-deficient nuclei are produced in more substantial quantities, so that many unstable proton-rich nuclei are produced. As already pointed out by Brancazio \& Cameron (1967), the irradiation of solar material by $\alpha$-particles favours the production of heavy nuclei, while a pure proton flux is globally destructive. This property is sensitive to the energy distribution of the accelerated particles. The low-energy component favours the radiative captures, while the high-energy one leads to transfer reactions with the possible emission of secondary particles. In particular, the higher the incident energy, the more the ( $\mathrm{p}$ or $\alpha, x \mathrm{n} y \mathrm{p} \mathrm{z} \alpha$ ) transfer reactions can be at the origin of a significant production of neutrons that, through their radiative neutron captures, tend to bring the flow back to the valley of $\beta$-stability. These secondary neutrons can play a crucial role, especially if we are concerned by the possible production of actinides. No por $\alpha$-captures could possibly lead to the synthesis of actinides or sub-actinides due to the low micro-second $\alpha$-unstable island on the $85 \leq Z \leq 89$ p-rich side of the valley of $\beta$-stability. Only a nuclear path on the neutron-rich side could be taken to produce transuranium significantly, and in this respect the production of secondary neutrons plays a decisive role. Particularly interesting is that the neutron density resulting from transfer reactions is rather independent of the value adopted for the local density $\rho$. Test calculations were performed for densities ranging between $\rho=10^{-9}$ to $1 \mathrm{~g} / \mathrm{cm}^{3}$, which indifferently lead to a constant product $\rho Y_{\mathrm{n}}$, that in first approximation can be expressed by

$\rho Y_{\mathrm{n}}=\frac{\sum_{k, x} x Y_{k}\left[\Phi_{\mathrm{p}}\langle\sigma\rangle_{\mathrm{p}, k}^{x}+\Phi_{\alpha}\langle\sigma\rangle_{\alpha, k}^{x}\right]}{\sum_{k} Y_{k} N_{a}\langle\sigma v\rangle_{\mathrm{n}}}$.

The resulting abundance distributions are therefore unaffected by changes in the local density. In other words, the impact of the neutron captures remains rather insensitive to the depth within the stellar atmosphere at which the spallation process takes place. All calculations shown here have been obtained for a local density of $\rho=10^{-7} \mathrm{~g} / \mathrm{cm}^{3}$.

\subsection{Power-law spectrum}

We here adopt the power law as the energy spectrum for the accelerated protons and $\alpha$-particles, which is given by Eq. (3) and consider different flux amplitudes, and power exponents, but restricting ourselves to $E_{\min }=1 \mathrm{MeV}$. We consider values of the 


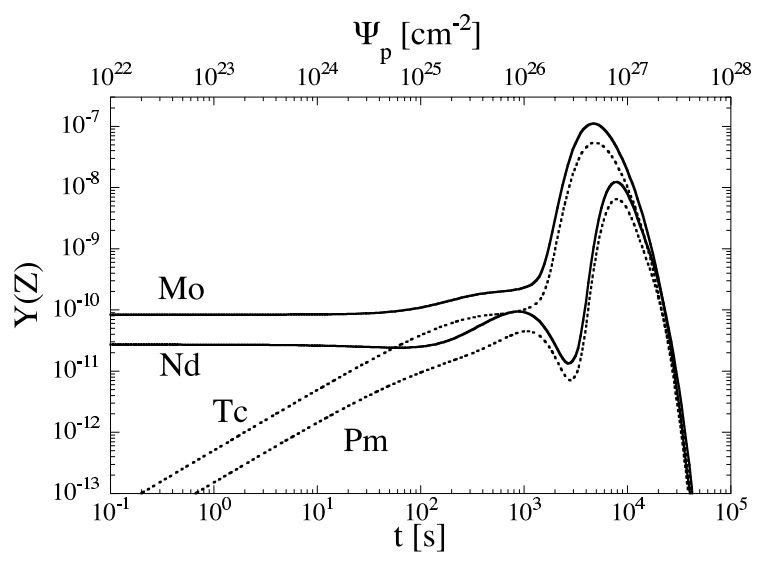

Fig. 1. Evolution with time $t$ or, equivalently, proton fluence $\Psi_{\mathrm{p}}$, of the Tc and Pm molar fractions, as well as their neighbour elements Mo and $\mathrm{Nd}$, respectively. The abundance evolution results from the irradiation by energetic particles corrresponding to $\Phi_{\mathrm{p}}=10^{-4} \mathrm{mb}^{-1} \mathrm{~s}^{-1}$, $\Phi_{\alpha} / \Phi_{\mathrm{p}}=0.1$, and a power-law spectrum with $\gamma=2$.

$\gamma$ exponent around 2 to 4 , as typically found in solar cosmic rays (e.g Lal 1972) and are used in models of either the early solar system (Leya et al. 2003) or of the interaction of energetic particles with interstellar matter (Ramaty et al. 1979). Proton fluxes between $\Phi_{\mathrm{p}}=10^{-8}$ and $1 \mathrm{mb}^{-1} \mathrm{~s}^{-1}$ are adopted with a relative $\alpha / p$ flux ratio ranging between 0.1 and 0.5. A first standard case corresponding to $\Phi_{\mathrm{p}}=10^{-4} \mathrm{mb}^{-1} \mathrm{~s}^{-1}, \Phi_{\alpha} / \Phi_{\mathrm{p}}=0.1$, and a power-law spectrum with $\gamma=2$ is studied in more detail.

In Fig. 1, we show the abundance evolution of the two radioelements Tc and Pm, as well as their neighbouring stable elements Mo and $\mathrm{Nd}$, respectively, as a function of time or, equivalently, the proton fluence $\Psi_{\mathrm{p}}=\int_{0}^{t} \Phi_{\mathrm{p}} \mathrm{d} t$. Minimum values of $10^{23} \mathrm{~cm}^{-2}$ are required to produce Tc and Pm in sufficient amounts to become observable (corresponding to a rough ratio of $\mathrm{Tc} / \mathrm{Mo}$ or $\mathrm{Pm} / \mathrm{Nd}$ of 0.01 ). Interestingly, a significant increase in all abundances is seen to take place for irradiation times longer than about $1000 \mathrm{~s}$, i.e. fluences higher than $10^{26} \mathrm{~cm}^{-2}$. At this time, $\alpha$-captures set in and drive the nuclear flow towards heavier species, transforming most of the light species into heavier ones. This is illustrated in Fig. 2 where the full elemental abundance distribution is compared with the solar and HD 101065 ones at four different irradiation times. The neutron density $N_{\mathrm{n}}$ reaches maximum values of $N_{\mathrm{n}}=10^{14} \mathrm{~cm}^{-3}$ for such fluences bringing the nuclear path back from the neutron-deficient side to the valley of $\beta$-stability. If fluences of the order $10^{26-27} \mathrm{~cm}^{-2}$ can be achieved, the abundances of the elements heavier than iron are seen to increase globally by 2 orders of magnitude. However, such conditions are not extreme enough to bridge the $N>126$ $\alpha$-unstable region between $\mathrm{Po}$ and Fr, so that no actinides are produced, the initial Th and $\mathrm{U}$ being in fact destroyed. For fluences higher than a few $10^{27} \mathrm{~cm}^{-2}$, the production of protons by the spallation reactions, essentially of $\alpha$-particles, dominates and reaches a stationary phase in which the molar fraction of all the heavy species decreases in time relative to the proton fraction (see Fig. 1, for the particular case of Mo, Tc, Nd, and Pm). The high-fluence abundance pattern is seen to be rather flat, since spallation reactions already at a few $\mathrm{MeV}$ per nucleon tend to smooth all nuclear structure effects out.

Obviously, this nucleosynthesis is quite sensitive to the parameter set adopted for the accelerated particles. We show in Fig. 3 the impact of the $\alpha$-particle flux on the abundance distribution obtained at large irradiation times $\tau_{\text {irr }}=10^{4} \mathrm{~s}$ for

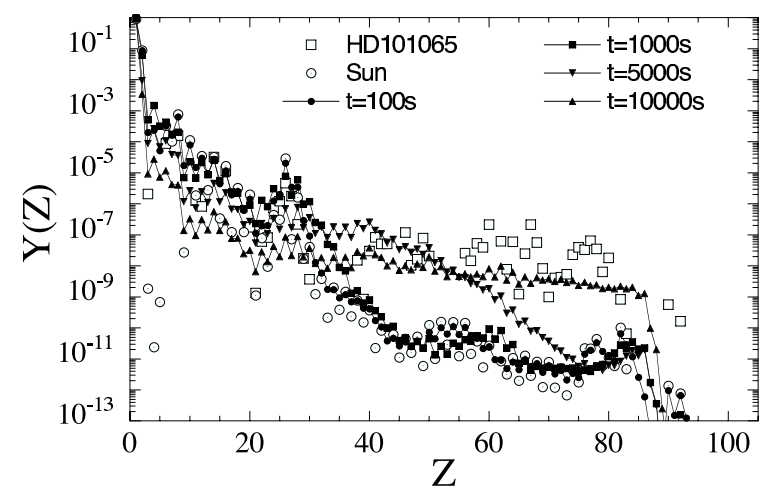

Fig. 2. Abundance distribution resulting from the irradiation process for $\Phi_{\mathrm{p}}=10^{-4} \mathrm{mb}^{-1} \mathrm{~s}^{-1}, \Phi_{\alpha} / \Phi_{\mathrm{p}}=0.1$, and a power-law spectrum with $\gamma=2$ at four different irradiation times $t$. For comparison, the HD 101065 abundances determined by Cowley et al. (2000) and the solar abundances taken as the initial composition are also plotted.

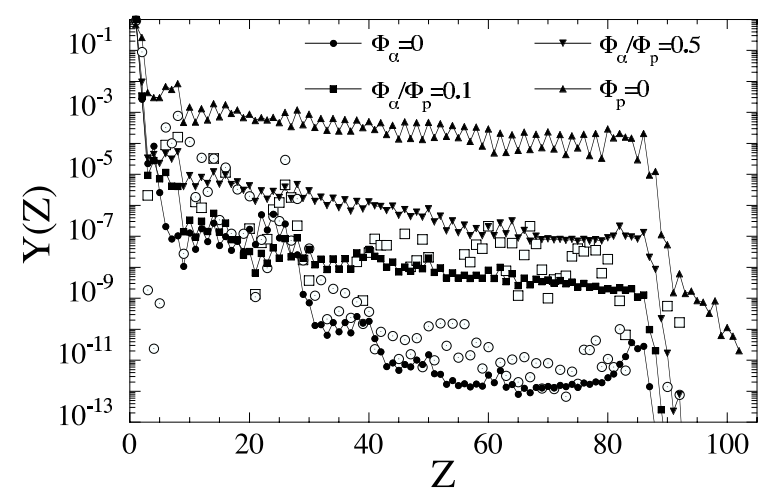

Fig. 3. Same as Fig. 2 for the abundance distribution resulting from the irradiation process for $\Phi_{\mathrm{p}}=10^{-4} \mathrm{mb}^{-1} \mathrm{~s}^{-1}, \gamma=2, \tau_{\text {irr }}=10^{4} \mathrm{~s}$, and for different values of the $\Phi_{\alpha} / \Phi_{\mathrm{p}}$ ratio. For the pure He irradiation, $\Phi_{\mathrm{p}}=0$ and $\Phi_{\alpha}=10^{-4} \mathrm{mb}^{-1} \mathrm{~s}^{-1}$.

different values of the $\Phi_{\alpha} / \Phi_{\mathrm{p}}$ ratio. While the most conventional value corresponds to 0.1 , the existence in the solar atmosphere of He-enriched regions with $\Phi_{\alpha} / \Phi_{\mathrm{p}}=0.5$ has been inferred from solar wind observations, as well as solar flare analysis (Mandzhavidze et al. 1997). Such an He-rich flux is seen in Fig. 3 to favour the late-time production of heavier species. The pure proton irradiation is totally inefficient for producing heavies, most of the species being in fact destroyed. In contrast, pure $\alpha$-irradiation is extremely efficient, all species from $\mathrm{Li}$ to $\mathrm{Bi}$ being produced at an average abundance level of about $10^{-3}$. A low, but significant synthesis of the sub-actinides and actinides is also found in the last case where its high value of $N_{\mathrm{n}}=10^{15} \mathrm{~cm}^{-3}$ leads the nuclear flow to the neutron-rich side of the valley of $\beta$-stability.

Similarly, if we adopt a steeper energy spectrum (see Fig. 4), the contribution from the high-energy $\alpha$-particles lowers, so that the $\alpha$-captures are less efficient for synthesizing the heavy species at late time. The neutron density reaches maximum values of $N_{\mathrm{n}}=4 \times 10^{12} \mathrm{~cm}^{-3}$ with the $\gamma=4$ spectrum of Fig. 4, i.e. a value about 25 times lower than in the $\gamma=2$ case (Figs. 2, 3). In this case, irradiation time scales that are about 10 times longer are needed to reach a stationary phase with a flat abundance pattern, as shown in Fig. 4.

We now compare the nucleosynthesis resulting from different flux amplitudes. Figure 5 shows the abundance distribution corresponding to proton fluxes of $\Phi_{\mathrm{p}}=10^{-8}, 10^{-4}$ and $1 \mathrm{mb}^{-1} \mathrm{~s}^{-1}$ for the same total fluence of $\Psi_{\mathrm{p}}=10^{27} \mathrm{~cm}^{-2}$ 


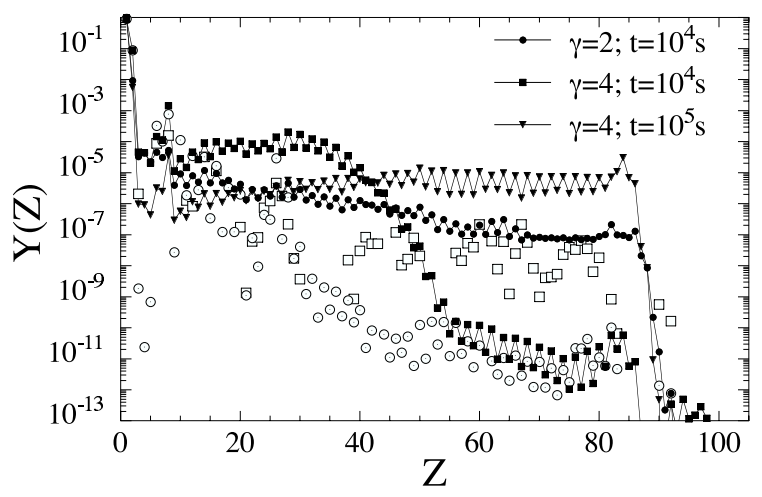

Fig. 4. Same as Fig. 2 for the abundance distribution resulting from the irradiation process for $\Phi_{\mathrm{p}}=10^{-4} \mathrm{mb}^{-1} \mathrm{~s}^{-1}, \Phi_{\alpha} / \Phi_{\mathrm{p}}=0.5$, and two values of $\gamma=2$ and 4 . The last case is given for two irradiation times, $\tau_{\text {irr }}=10^{4} \mathrm{~s}$ and $10^{5} \mathrm{~s}$.

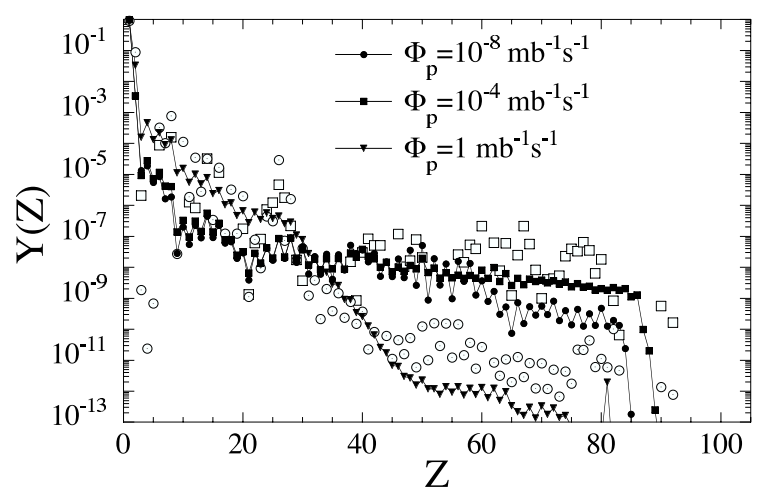

Fig. 5. Same as Fig. 2 for the abundance distribution resulting from the irradiation process for three different values of the proton flux $\Phi_{\mathrm{p}}$ and $\Phi_{\alpha} / \Phi_{\mathrm{p}}=0.1, \gamma=2$, and a total fluence $\Psi_{\mathrm{p}}=10^{27} \mathrm{~cm}^{-2}$.

(corresponding to irradiation times $\tau_{\text {irr }}=10^{8}, 10^{4}$ and $1 \mathrm{~s}$, respectively) and the same $\Phi_{\alpha} / \Phi_{\mathrm{p}}=0.1$ and $\gamma=2$ values. The production of heavy nuclei resulting from the $\Phi_{\mathrm{p}}=$ $10^{-8} \mathrm{mb}^{-1} \mathrm{~s}^{-1}$ irradiation is seen to be less efficient, for the same total fluence, than the one obtained with $\Phi_{\mathrm{p}}=10^{-4} \mathrm{mb}^{-1} \mathrm{~s}^{-1}$. In the first case, the $\alpha$-captures take place on much longer time scales (of the order of a year), so that both $\beta$ - and $\alpha$-decays become significant competitors in the reaction flows. In contrast, for large flux amplitudes $\left(\Phi_{\mathrm{p}}=1 \mathrm{mb}^{-1} \mathrm{~s}^{-1}\right)$, the nuclear flow is moved strongly to the p-rich side where the spallation reactions with proton- and $\alpha$-emission dominate (due to the high energies encountered in the flat $\gamma=2$ spectrum), and most of the matter is recycled back into light species. However, it should be stressed that the present conclusion only holds for the adopted energy spectrum and $\alpha$-to-p flux ratio (see Sect. 3.2).

\subsection{Energy-constant spectra}

We now turn to the scenario in which the energy distribution of the accelerated particles is assumed to be constant in a relatively narrow energy range (typically 5 to $10 \mathrm{MeV}$ ) corresponding to a given energy spread in the acceleration process. We first concentrate on the constant energy distribution in the $0 \leq E \leq 10 \mathrm{MeV}$ range.

In Fig. 6, we show the evolution of Tc, Mo, Pm, and $\mathrm{Nd}$ as a function of time or, equivalently, of the proton fluence. As in Fig. 1, minimum values of $10^{23} \mathrm{~cm}^{-2}$ are required to synthesize Tc and $\mathrm{Pm}$ at a level high enough to become observable. In

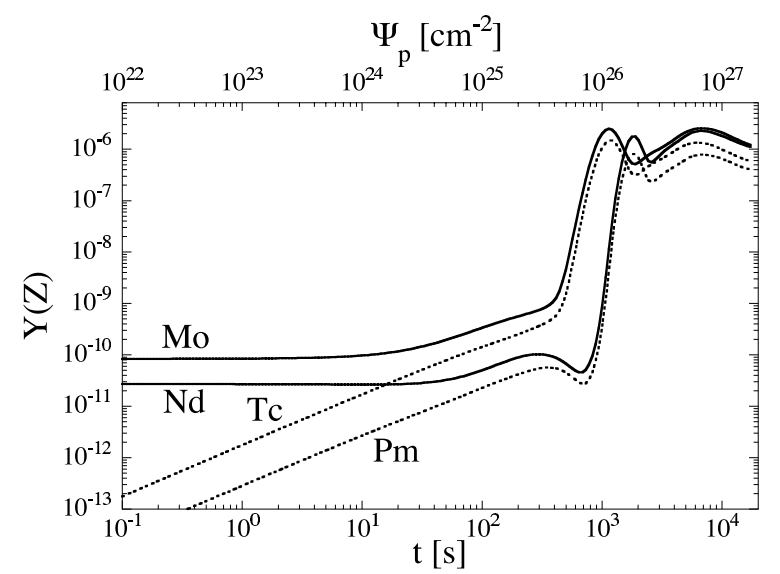

Fig. 6. Evolution with time $t$ or, equivalently, proton fluence $\Psi_{\mathrm{p}}$, of the Tc and Pm molar fractions, as well as their neighbour elements Mo and $\mathrm{Nd}$, respectively. The abundance evolution results from the irradiation by energetic particles corrresponding to $\Phi_{\mathrm{p}}=10^{-4} \mathrm{mb}^{-1} \mathrm{~s}^{-1}, \Phi_{\alpha} / \Phi_{\mathrm{p}}=$ 0.1 , and $E_{\max }=10 \mathrm{MeV}$.

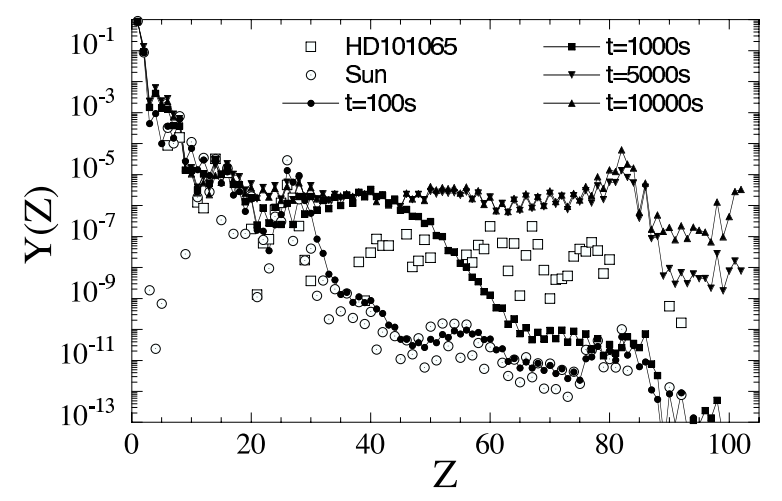

Fig. 7. Abundance distribution resulting from the irradiation process for $\Phi_{\mathrm{p}}=10^{-4} \mathrm{mb}^{-1} \mathrm{~s}^{-1}, \Phi_{\alpha} / \Phi_{\mathrm{p}}=0.1$, and an energy-constant spectrum with $E_{\min }=0 \mathrm{MeV}$ and $E_{\max }=10 \mathrm{MeV}$ at four different irradiation times. For comparison, the HD 101065 abundances determined by Cowley et al. (2000) and the solar abundances taken as initial composition are also plotted.

contrast to the power-spectrum case, at extreme fluences of the order of $10^{27} \mathrm{~cm}^{-2}$, the abundances do not drop with increasing irradiation. A relatively stationary phase is achieved in which the abundances of all the heavy elements remains relatively constant in time, as also shown in Fig. 7, where the full elemental abundance distribution is compared with the solar and HD 101065 ones for four different irradiation times. In this specific scenario, if fluences of the order $10^{26-27} \mathrm{~cm}^{-2}$ can be achieved, can the abundances of the elements heavier than iron not only be increased by 5 orders of magnitude, but also the neutron flux becomes strong enough to bridge the $N>126 \alpha$-unstable region between $\mathrm{Po}$ and $\mathrm{Fr}$ and produce actinides with a charge as high as $Z \simeq 100$ in large amounts. This is essentially due to the high neutron densities of $N_{\mathrm{n}} \simeq 10^{15} \mathrm{~cm}^{-3}$ reached under these specific conditions. Indeed, for energies around 5 to $10 \mathrm{MeV}$ per nucleon, the dominant reactions are $\alpha$-transfer reactions with one to three neutron emission. The nuclear flow at an irradiation time greater than some $1000 \mathrm{~s}$ is shifted to the neutron-rich side of the valley of stability. This property has the decisive effect of enabling a significant production of actinides. Such conditions could not be achieved with the power-law spectrum due to the weak contribution of these $(5-10 \mathrm{MeV})$ energies with respect to the lowest ones $\left(E_{\min }=1 \mathrm{MeV}\right.$ was adopted in the case 


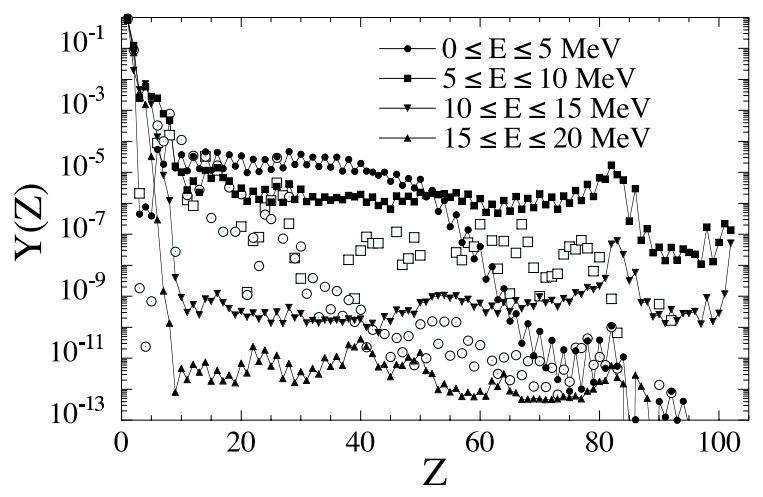

Fig. 8. Same as Fig. 7 for the abundance distribution resulting from the irradiation process for $\Phi_{\mathrm{p}}=10^{-4} \mathrm{mb}^{-1} \mathrm{~s}^{-1}, \Phi_{\alpha} / \Phi_{\mathrm{p}}=0.1, \tau_{\text {irr }}=5000 \mathrm{~s}$, and four energy-constant spectra as given in the legend.

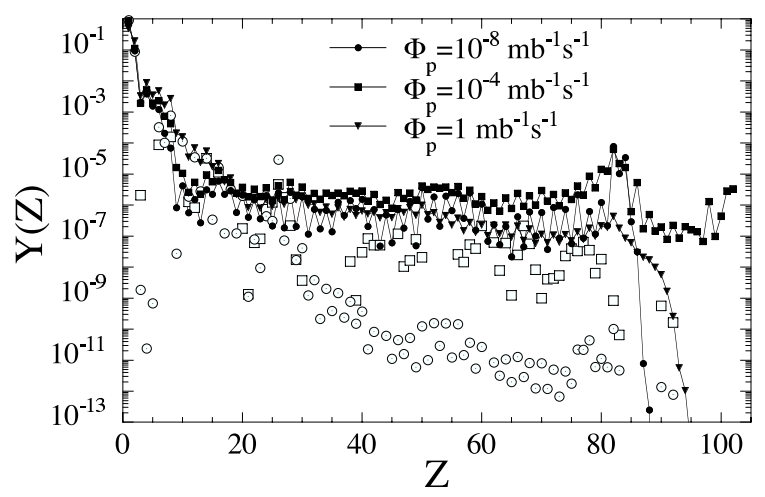

Fig. 9. Same as Fig. 7 for the abundance distribution resulting from the irradiation process for three different values of the proton flux $\Phi_{\mathrm{p}}$ and $\Phi_{\alpha} / \Phi_{\mathrm{p}}=0.1$, but the same total proton fluence of $\Psi_{\mathrm{p}}=10^{27} \mathrm{~cm}^{-2}$. The energy-constant spectrum with $E_{\min }=0 \mathrm{MeV}$ and $E_{\max }=10 \mathrm{MeV}$ is adopted

studies of Sect. 3.1). This is also illustrated in Fig. 8 where different energy regimes are considered for the same irradiation time. For low energies $(0-5 \mathrm{MeV})$ at very high fluences, the heaviest elements have not been produced yet, higher fluences being required to produce the $\mathrm{Pb}-\mathrm{Bi}$ peak elements. The nuclear flow in this specific case remains unable to bridge the Po-Fr instablility island. The 5-10 MeV energy range is seen to be the most efficient regime for producing the heavy elements (at least in the specific conditions adopted in Fig. 8), the final distribution being almost identical to the one obtained in Fig. 7. If $\alpha$-particles with higher energies are considered (see Fig. 8), multi-particle transfer reactions dominate and tend to recycle the material back to light species.

For less intense proton and $\alpha$-particle fluxes, the production of the heavy elements up to $\mathrm{Pb}$ remain relatively similar at a given total fluence (Fig. 9). However, the maximum neutron densities achieved are significantly lower (about 4 orders of magnitude between the $\Phi_{\mathrm{p}}=10^{-8}$ and $10^{-4} \mathrm{mb}^{-1} \mathrm{~s}^{-1}$ cases shown in Fig. 9), so that the production of superheavies through a neutron-rich path becomes impossible. In the $\Phi_{\mathrm{p}}=1 \mathrm{mb}^{-1} \mathrm{~s}^{-1}$ case shown in Fig. 9, $N_{\mathrm{n}}$ reaches values of $10^{16} \mathrm{~cm}^{-3}$, and the flow is shifted deep into the neutron-rich region, with the result that $\beta^{-}$decays become more efficient and $\alpha$-captures less effective. Transuranium nuclei are still produced. Note that in this scenario, like in the one corresponding to the power-law spectrum discussed in Sect. 3.1 (see Fig. 4 in particular), the nucleosynthesis of the elements heavier than iron is favoured by an increase in the $\alpha$-to-p flux ratio. The final high-fluence abundance pattern is also flat, since the capture of charged particles with energies higher than a few $\mathrm{MeV}$ per nucleon inevitably smooth most of the nuclear structure effects out.

\section{Comparison with HD 101065 surface abundances}

From the general study of Sect. 3, the present nucleosynthesis turns out to be attractive in many respects to explain the abundance estimated at the surface of the CP star HD 101065. First of all, it can be held responsible for a significant production of elements heavier than iron by a few orders of magnitude, without having to call for additional diffusive processes. This nucleosynthesis can be accompanied by a significant production of radioelements, not only Tc or Pm, but also actinides ranging from Po to Fm, at least for the extreme conditions discussed in Sect. 3.2.

As far as a quantitative comparison is concerned, the irradiation process leads to structureless abundance pattterns, and so it cannot, as such, account for the abundance distribution of HD 101065 (see Figs. 2-9). It should be stressed here that two nucleosynthesis effects, which have not been discussed so far, tend to modify this statement. The first one concerns the possible neutron capture that may still take place after the irradiation. If the neutron density is high enough at the time of the irradiation freeze-out, neutrons keep on being captured (and at low temperatures, the neutron capture rate is strongly affected by nuclear structure properties), so that some nuclear structure effect may be recovered in the final distribution. Second, at the time of observation, the unstable nuclei return to the valley of stability by $\beta$ - and/or $\alpha$-decays, leading to possible imprints of nuclear origin. Note, however, that if we assume that $\mathrm{Pm}$ in particular is still present in the atmosphere of HD 101065, the time elapsed between the nucleosynthesis and the observation cannot be much longer than a few years.

In the present scenario, there is no reason to believe that the total atmosphere of HD 101065 has been irradiated by energetic particles with similar properties. We do not study possible superpositions of different events at this stage because of the already large parameter space being spanned. In turn, we can reasonably assume that the material irradiated for a given time $\tau_{\text {irr }}$ is mixed (in a way or another) with unaffected surrounding layers of solartype composition or, equivalently, that only part of the observed material has been irradiated. In this case, the final abundances can be estimated by

$Y_{(Z, A)}^{\operatorname{mix}}=f_{\text {mix }} Y_{(Z, A)}^{\mathrm{irr}}+\left(1-f_{\text {mix }}\right) Y_{(Z, A)}^{\odot}$

where $f_{\text {mix }}$ is an arbitrary dilution factor.

If we consider one of the specific irradiation events studied in Sect. 3.2 corresponding to the parameter set of $\Phi_{\mathrm{p}}=$ $10^{-4} \mathrm{mb}^{-1} \mathrm{~s}^{-1}, \Phi_{\alpha} / \Phi_{\mathrm{p}}=0.1, \tau_{\text {irr }}=5000 \mathrm{~s}$, and an energyconstant spectrum with $0 \leq E \leq 10 \mathrm{MeV}$, we see in Fig. 10 that globally the observed abundances can be explained remarkably well, including the presence of Tc, Pm, and transuranium. A dilution factor of $f_{\text {mix }}=0.02$ is adopted to reproduce the global overabundance of HD 101065 heavy elements of 3-4 dex with respect to the solar values. All details of the observed abundance distribution, and in particular structure effects for elements heavier than iron, are not reproduced, but this can be due, for example, to the relatively approximate treatment of the neutron capture.

Figure 10 illustrates a specific irradiation event. The HD 101065 abundances can, however, be explained by events 


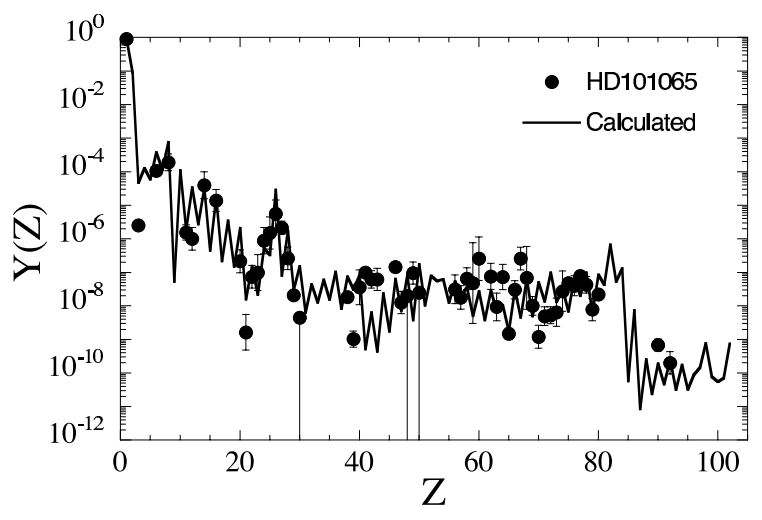

Fig. 10. Comparison of HD 101065 and calculated abundances. The calculated abundances result from the mixing of solar abundances with irradiated matter. The irradiation fluxes are characterised by $\Phi_{\mathrm{p}}=$ $10^{-4} \mathrm{mb}^{-1} \mathrm{~s}^{-1}, \Phi_{\alpha} / \Phi_{\mathrm{p}}=0.1, E_{\max }=10 \mathrm{MeV}$, and an irradiation timescale of $\tau_{\text {irr }}=5000 \mathrm{~s}$. The distribution is evolved up to a time $t=10^{4} \mathrm{~s}$ before being mixed with the solar composition by a dilution factor of $f_{\text {mix }}=0.02$. The full dots correspond to the HD 101065 abundance determination of Cowley et al. (2000).

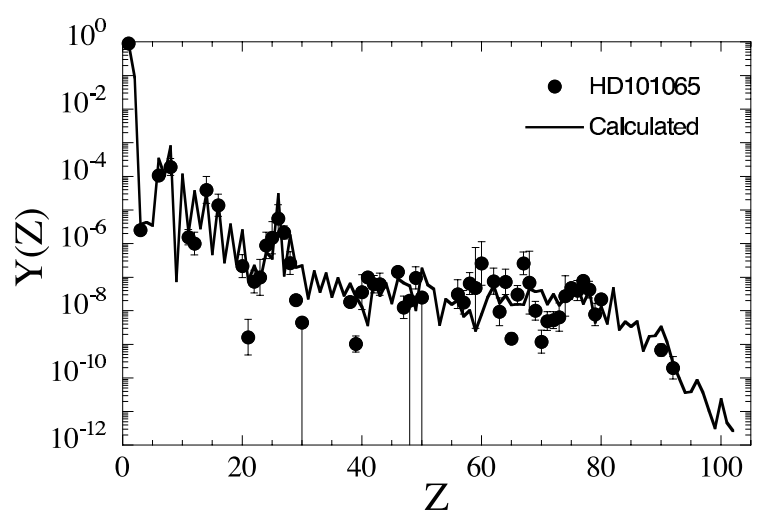

Fig. 11. Same as Fig. 10 for irradiation fluxes characterised by $\Phi_{\mathrm{p}}=0$, $\Phi_{\alpha}=10^{2} \mathrm{mb}^{-1} \mathrm{~s}^{-1}, E_{\max }=10 \mathrm{MeV}, \tau_{\text {irr }}=1 \mathrm{~ms}, \rho=10^{-3} \mathrm{~g} / \mathrm{cm}^{-3}$ and a dilution factor of $f_{\text {mix }}=0.0005$.

with very different characteristics. As a second example, we show in Fig. 11 the final abundance distribution obtained after mixing solar-like material with matter exclusively irradiated by $0 \leq E \leq 10 \mathrm{MeV} \alpha$-particles for about $1 \mathrm{~ms}$ at a rate of $10^{2} \mathrm{mb}^{-1} \mathrm{~s}^{-1}$. The density of the irradiated material is taken to be $\rho=10^{-3} \mathrm{~g} / \mathrm{cm}^{-3}$, so that the neutron thermalization can be achieved on shorter timescales than the nucleosynthesis one. Here again, globally the observed abundance distribution is quantitatively well reproduced, and even the $\mathrm{Li}$ abundances agree. Some details are still missing, for example $\mathrm{Sc}, \mathrm{Cu}, \mathrm{Zn}$, or $\mathrm{Y}$ abundances are overestimated. The observed $\mathrm{Yb}$ underabundance and the resulting inverse odd-even effect around $\mathrm{Yb}$, often considered a signature of diffusion processes, cannot be explained by the present calculations either.

Finally, HD 101065 is also characterised by abnormally high $\mathrm{Co} / \mathrm{Fe}$ and ${ }^{6} \mathrm{Li} /{ }^{7} \mathrm{Li}$ ratios. Among the light elements, $\mathrm{Co}$ is indeed identified as having the most abnormal abundance, which differs from the solar value by $1.6 \mathrm{dex}$, while for the other light elements, the average departure from solar is only $0.1 \mathrm{dex}$ (Cowley et al. 2000). We compare the observed ratios in Fig. 12 with the fluence-dependent values calculated in the specific scenario developed in Sect. 3.2. Again, for high fluences of about $\Psi_{\mathrm{p}}=10^{26-27} \mathrm{~cm}^{-2}$, both calculated ratios are seen to be compatible with observation, which is a new and interesting result that

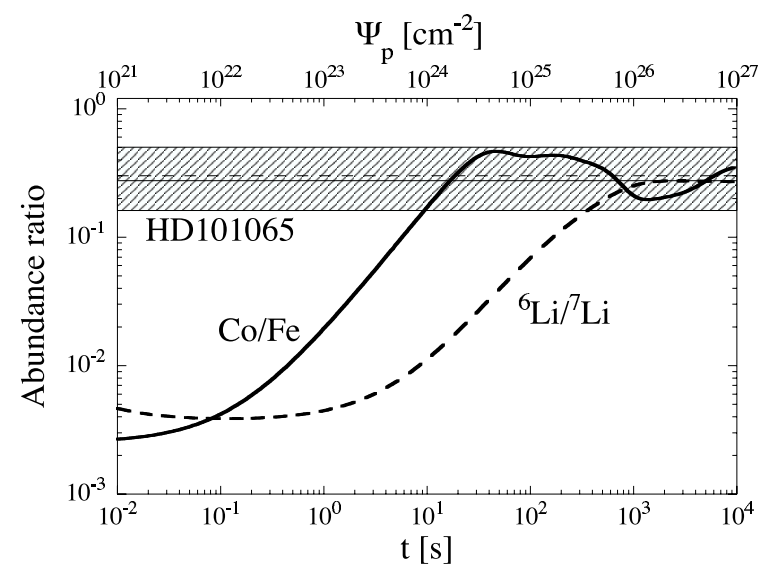

Fig. 12. Abundance ratio for $\mathrm{Co} / \mathrm{Fe}$ (solid) and ${ }^{6} \mathrm{Li} /{ }^{7} \mathrm{Li}$ (dashed line) as a function of time (or proton fluence) resulting from the irradiation process with $\Phi_{\mathrm{p}}=10^{-4} \mathrm{mb}^{-1} \mathrm{~s}^{-1}, \Phi_{\alpha} / \Phi_{\mathrm{p}}=0.1$, and an energy-constant spectrum with $E_{\min }=0 \mathrm{MeV}$ and $E_{\max }=10 \mathrm{MeV}$. This irradiated material is not mixed with solar-like material. For comparison, the $\mathrm{Co} / \mathrm{Fe}$ ratio determined at the HD 101065 surface is given by the thin solid horizontal line and the corresponding error box of 0.2 dex around (Cowley et al. 2000). The value of the ${ }^{6} \mathrm{Li} /{ }^{7} \mathrm{Li}=0.3(0.2-0.6)$ isotopic ratio determined by Shavrina et al. $(2003,2006)$ is shown by the horizontal thin dashed line.

could favour the nuclear irradiation process as the one responsible for the abnormal HD 101065 composition.

In summary, many spectroscopic observations of HD 101065 can be met if we assume that extremely high proton and $\alpha$-particle fluences have irradiated solar-like material. This requires, however, fluences that are much higher than what would be expected in normal or even extreme stellar flares, as discussed in Sect. 1. The exposure parameter $w$ introduced in Sect. 2 can also be estimated for the two events considered in Figs. 10, 11. It amounts to $8 \times 10^{11}$ and $2 \times 10^{11} \mathrm{erg} \mathrm{s} \mathrm{cm}^{-3}$, respectively, i.e. a value comparable or even lower than the $10^{13} \mathrm{erg} \mathrm{s} \mathrm{cm}^{-3}$ determined by Brancazio \& Cameron (1967) to explain the overabundances in CP A-stars. Following the same qualitative argument as Brancazio \& Cameron (1967), if the particle acceleration were to take place in a magnetic flux tube of length $L \simeq 10^{9} \mathrm{~cm}$, this process would require flares with a magnetic field $H>\sqrt{8 \pi \omega v / L} \simeq 10^{6} \mathrm{G}$, i.e. a 1000 times stronger than the stellar magnetic field. This intense field cannot be excluded in stellar flares. However, the irradiation event considered here to explain the production of actinides is rather intense and short. The energy flux $F=\phi\langle E\rangle \simeq 8 \times 10^{17}$ and $8 \times 10^{23} \mathrm{erg} \mathrm{cm}^{-2} \mathrm{~s}^{-1}$ for the event shown in Figs. 10, 11, respectively. These are by orders of magnitude higher than the radiant flux $\sigma T_{\mathrm{e}}^{4} \simeq 10^{11} \mathrm{erg} \mathrm{cm}^{-2} \mathrm{~s}^{-1}$, which casts doubt on the possibility of finding such short and intense acceleration in the stellar atmosphere (see Cowley 1973, for a more detailed discussion).

The possible existence of these irradiation events needs to be confirmed by hydrodynamical simulations, but most of all by spectroscopic observations through the detection of short-lived radioelements. If these observations clearly indicate the need to introduce high-fluence irradiations, theoretical astrophysics will face a new challenge, relatively similar to the one faced by the r-process nucleosynthesis, i.e. to identify the relevant astrophysical sites in which these extreme conditions could be met. 


\section{Conclusion}

The present study has aimed at exploring spallation nucleosynthesis as a possible explanation of the peculiar abundances spectroscopically determined at the surface of HD 101065. Due to the unknown characteristics of the accelerated particles that would be held responsible for the nucleosynthesis process, a purely parametric approach was followed, taking as free parameters the proton and $\alpha$-particle flux amplitude and energy distribution, the time of irradiation, and the possible mixing with unaffected material. We have shown that at least the specific simulations shown here with high fluences tend to explain many different observational aspects, qualitatively as well as quantitatively. This includes, in particular, a significant production of $Z>30$ heavy elements through radiative captures and spallation reactions, but also a significant synthesis of radioelements, such a Tc, $\mathrm{Pm}$, and actinides up to $Z \simeq 100$. Even the observed $\mathrm{Co} / \mathrm{Fe}$ and ${ }^{6} \mathrm{Li} /{ }^{7} \mathrm{Li}$ abundance ratios can be matched.

Obviously, improvements still need to be made in the parametric modelling, in particular a better description of the neutron capture rate at low temperatures and a full survey of the large parameter space characterising the property of the accelerated particles. Realistic models describing the acceleration process and the huge fluences required need to be developed to make this scenario a viable explanation.

Even if such a process could meet many observational aspects, the diffusion process has proven, at least qualitatively, to be important for understanding the atmosphere of CP stars. It will remain difficult to disentangle the effects of both processes theoretically. The contribution of the nuclear process would be strengthened if spectroscopic observation could confirm the presence of short-lived radioelements on the surface of CP stars and, if confirmed, provide abundance determination for these clear tracers of nuclear activity, hopefully in the near future.

\section{References}

Arnould, M., \& Goriely, S. 2006, Nucl. Phys. A, 777, 157

Bidelman, W. P. 2005, in Proc. ASP Conf. Ser. 336, ed. T. G. Barnes, \& F. N. Bash, 309

Brancazio, P. J., \& Cameron, A. G. W. 1967, Can. J Phys., 45, 3297

Clayton, D., Dwek, E., \& Woosley, S. E. 1977, ApJ, 214, 300

Cowley, C. R. 1973, Observatory, 93, 195

Cowley, C. R., Ryabchikova, T., Kupka, F., et al. 2000, MNRAS, 317, 299

Cowley, C. R., Bidelman, W. P., Hubrig, S., Mathys, G., \& Bord, D. J. 2004, A\&A, 419, 1087

Cowley, C. R., \& Bord, D. J. 2004, in Proc. IAU Symp. 224 (Cambridge University Press), ed. J. Zverko et al., 265

Cowley, C. R. 2005, Phys. Scr., T119, 26

Fujimoto, S., Kotake, K., Yamada, S., Hashimoto, M., \& Sato, K. 2006, ApJ, 644, 1040

Gopka, V. F., Yushchenko, A. V., Shavrina, A. V., et al. 2004, in Proc. IAU Symp. 224, ed. J. Zverko et al., 734

Gopka, V. F., Yushchenko, A. V., Goriely, S., Shavrina, A. V., \& Kang, Y. W. 2006, in AIP Conf. Ser. 847, ed. Kubono et al., 389

Koning, A. J., Hilaire, S., \& Duijvestijn, M. C. 2005, Proc. Int. Conf. on Nuclear Data for Science and Technology, ed. Haight C. et al., AIP Conf., 769, 1154

Kurtz, D. W. 1982, MNRAS, 200, 807

Lal, D. 1972, Spac. Sci. Rev., 14, 3

Leya, I., Halliday, A. N., \& Wieler, R. 2003, ApJ, 594, 605

Maeda, K., \& Nomoto, K. 2003, ApJ, 598, 1163

Mandzhavidze, N., Ramaty, R., \& Kozlovsky, B. 1997, ApJ, 489, L99

Meyer, J. P. 1972, ApJS, 7, 417

Michaud, G. 1970, ApJ, 160, 641

Michaud, G. 2004, in Proc. IAU Symp. 224, ed. J. Zverko et al. (Cambridge University Press), 173

Ramaty, R., Kozlovsky, B., \& Lingenfelter, R. E. 1979, ApJS, 40, 487

Padovani, P. 2004, in Proc. IAU Symp. 224, ed. J. Zverko et al. (Cambridge University Press), 485

Shavrina, A. V., Polosukhina, N. S., Pavlenko, Ya. V., et al. 2003, A\&A, 409, 707

Shavrina, A. V., Polosukhina, N. S., Pavlenko, Ya. V., et al. 2006, Astron. Rep., 50,500

Zverko, J., Ziznovsky, J., Adelman, S. J., \& Weiss, W. W. 2004, The A-star Puzzle, Proc. IAU Symp., 224 (Cambridge University Press) 